\title{
Idiopathic Hypertrophic Spinal Pachymeningitis: A Diagnostic Challenge: A Case Report and Review of the Literature
}

\author{
Abdulla Alsulaiman ${ }^{1,2}$ \\ ${ }^{1}$ Department of Neurology, College of Medicine, Imam \\ Abdulrahman Bin Faisal University, Dammam, Kingdom of \\ Saudi Arabia \\ ${ }^{2}$ Department of Neurology, King Fahd Hospital of the University, \\ Al Khobar, Kingdom of Saudi Arabia \\ J Neurosci Rural Pract 2020;11:175-177
}

\begin{abstract}
Keywords

- dural thickening

- idiopathic hypertrophic spinal pachymeningitis

- magnetic resonance imaging

- myelopathy
\end{abstract}

\author{
Address for correspondence Abdulla Alsulaiman, Department \\ of Neurology, King Fahd Hospital of the University, PO Box 2208, \\ Al Khobar, Kingdom of Saudi Arabia (e-mail: aalsulaiman@iau.edu.sa).
}

\section{Introduction}

Idiopathic hypertrophic pachymeningitis (IHP) is an extremely rare disorder distinguished by long-standing nonspecific granulomatous inflammation of the dura with fibrosis that can result in compression of the brain or spinal cord. ${ }^{1,2}$ Idiopathic hypertrophic spinal pachymeningitis (IHSP) is often diagnosed by exclusion supported by classical magnetic resonance imaging (MRI) features and confirmed with histopathological examination of a biopsy specimen of the dura mater. ${ }^{1}$ The mainstay of treatment is medical treatment (i.e., corticosteroids). However, if it does not respond, then surgical decompression may be needed to prevent neurological sequelae. ${ }^{3}$

\section{Case Report}

A 38-year-old female patient presented to us with neck pain and progressive quadraparesis. Her pain started 2 years before this visit and was described as progressive and continuous in nature, radiating to the upper limbs and back. The patient denied any history of headache, blurred vision, urine or stool incontinence, joint pain, skin rash, morning stiffness, or recent trauma. She had no past history of tuberculosis contact. Family and past history for this patient were unremarkable. She was initially diagnosed as having a subacute epidural hematoma in another hospital and was treated with intravenous corticosteroids. Postcorticosteroid treatment, her symptoms improved and the patient was subsequently discharged home. Later on, when her symptoms recurred, she was brought to our hospital. On examination, the patient was alert, attentive, and oriented. Cranial nerves were intact and fundoscopic examination was normal. The tone was increased in both upper and lower limbs and power was four-fifths, bilaterally in the upper and lower limbs. Deep tendon reflexes were plus three and symmetrical at the biceps, triceps, knees, and ankles with a positive Hoffman's sign, bilaterally. Plantar responses were equivocal bilaterally. A sensory level was noted at T4. 
Laboratory investigations included routine blood tests and C-reactive protein, erythrocyte sedimentation rate, rheumatoid factor, antinuclear antibody, double-stranded DNA, antiphospholipid antibody, lupus anticoagulant, antineutrophil cytoplasmic antibodies (p-ANCA and c-ANCA), Ro (SS-A), La (SS-B), U1RNP, Jo-1, and anticyclic citrullinated peptide (CCP) antibody levels. All were noted to be within normal range. A tuberculin skin test was negative. Serologic investigations of HIV and VDRL were also negative. Serum angiotensin-converting enzyme levels were found to be normal. A lumbar puncture was performed. Cerebrospinal fluid (CSF) analysis showed an elevated white blood cell count with predominant lymphocyte reaching 80 with protein as $62 \mathrm{mg} / \mathrm{dL}$ and normal sugar. Special stains for bacteria, mycobacteria, and fungi tests were negative. CSF tuberculosis antibody test was also negative.

A spinal MRI was done. The sagittal view displayed predominant thickened anterior and posterior dura to the patient's cord from the $\mathrm{C} 2$ to $\mathrm{C} 7$ spine level with hypointense T2 signal with peripheral contrast enhancement on T1-weighted gadolinium-enhanced images ( - Fig. 1A, B).

Confirmatory biopsy was eventually performed. The microscopic examination showed fibrous collagenized tissue with hyalinization and infiltration of chronic inflammatory cells, mainly lymphocytes, without any evidence of malignancy. Finally, a diagnosis of IHP was made based on the evidence provided above.

The patient was started on methylprednisolone pulse therapy ( $1 \mathrm{~g}$ daily) for a total of 5 days. After that, she received a maintenance of oral prednisone of $50 \mathrm{mg} / \mathrm{day}$ for a total of 2 weeks. After this regimen, the patient improved significantly, both clinically and radiologically ( - Fig. 2 ).

At the 2-year follow-up, the patient reported another relapse of her symptoms, and thus another course of corticosteroids was initiated with improvement.

\section{Discussion}

Hypertrophic pachymeningitis (HP) is a rare disorder with nonspecific symptoms of myelopathy that are often dependent on its anatomical location. Underreporting of such cases is more likely due to its nonspecific presentation

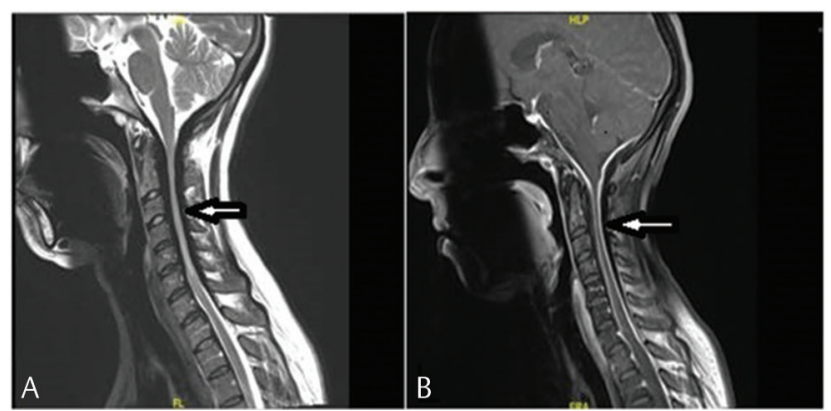

Fig. 1 (A) Sagittal T2 magnetic resonance imaging of the cervical spine showing ventral and dorsal thickening of the dura mater with compression of the spinal cord from C2 to C7 spine level. (B) Sagittal T1 gadolinium-enhanced magnetic resonance imaging of the cervical spine showing enhancement of the meninges at the cervical part of the spinal cord. HLP, hypertrophic pachymeningitis.

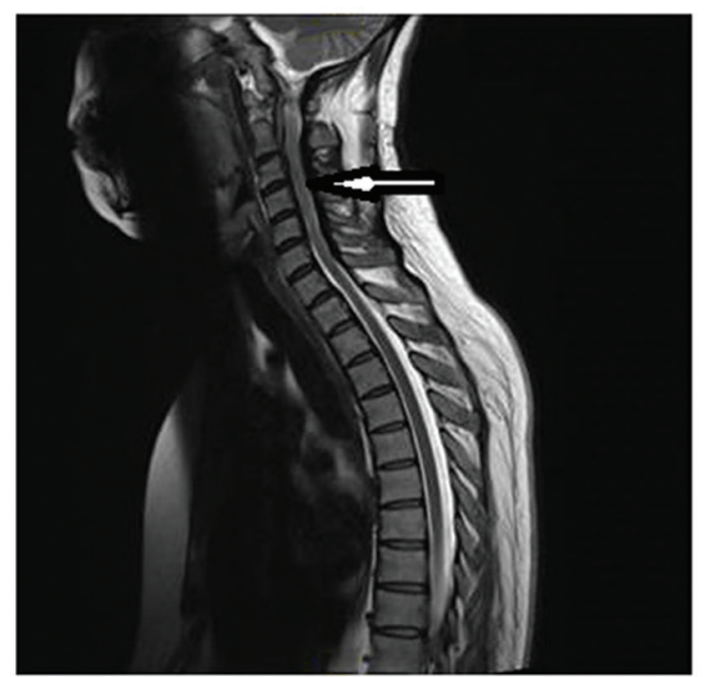

Fig. 2 Sagittal T2 magnetic resonance imaging of the cervical spine showing improvement in the thickening of the dura mater after steroid therapy.

and chronic progression. Chronic HP of the spine is caused by inflammation of the dura mater. Typically, it involves the cervical and thoracic spine areas. ${ }^{4}$

Variations in clinical presentation are not only depended on the anatomic level of presentation but also on clinical progression as well. HP is classified according to its etiology as idiopathic and secondary. Secondary types may include etiologies, such as trauma, metabolic disorder, autoimmune disease, neoplasms, sarcoidosis, and infectious diseases, such as neurosyphilis, tuberculosis, human T-lymphotropic virus-1, meningococcal meningitis, and fungal infections. ${ }^{5}$ Therefore, excluding underlying secondary etiologies is considered an extensive process which may alter the management and disease progression of this disorder.

In this case, we explored all possible causative factors but failed to identify any underlying or associated cause and the diagnosis of IHP was made. Until now, the pathophysiology of IHP remains unclear, with some studies inferring that aberration and unregulated autoimmune response may be the cause, especially since most cases of IHP are symptomatically improved with corticosteroids and other immunosuppressant agents, such as cyclophosphamide, azathioprine, and methotrexate when corticosteroid resistance is seen. ${ }^{6}$

MRI is the best diagnostic imaging modality for such cases. In particular, gadolinium-enhanced MRIs are preferred. However, despite good clinical response to medical management, abnormal dural enhancement may still be evident on MRI. ${ }^{7}$

The gold standard diagnostic test for such cases is an open biopsy of the thickened dura mater. However, biopsy would most often identify nonspecific dural inflammatory changes, including plasma cells, giant cells, neutrophils, eosinophils, and lymphocytes. ${ }^{8}$

Although IHP management is controversial, surgical decompression is often performed. This provides both rapid symptomatic relief and prevents serious neurological sequelae. Most cases of IHP show radical improvement and less susceptibility to relapse when combining both 
surgical decompression and postoperative pulse steroids. Some studies have proposed other treatment options such as radiotherapy, methotrexate, cyclophosphamide, or empirical antituberculous therapy. Nevertheless, the effectiveness of such therapies remains unclear. ${ }^{1}$ Some authors suggest supplementing with methotrexate or azathioprine in the event the patient symptoms reappear or relapse after they have tapered their doses of corticosteroids. ${ }^{7}$

\section{Conclusion}

Our main purpose of presenting this case report was to demonstrate the complex diagnostic dilemma with IHP diagnosis. Close patient follow-up must be conducted to help confirm the diagnosis. We posit that more studies need to be conducted in this area that include randomized control trials as a viable means to help develop guidelines for appropriate treatment management of IHP.

\section{Conflict of Interest}

None declared.

\section{Acknowledgment}

This research made use of the computational resources and technical services provided by the Scientific and High-Performance Computing Center at Imam Abdulrahman Bin Faisal University.

\section{References}

1 Qin LX, Wang CY, Hu ZP, Zeng LW, Tan LM, Zhang HN. Idiopathic hypertrophic spinal pachymeningitis: a case report and review of literature. Eur Spine J 2015;24(Suppl 4):S636-S643

2 Zhu R, He Z, Ren Y. Idiopathic hypertrophic craniocervical pachymeningitis. Eur Spine J 2015;24(Suppl 4):S633-S635

3 Ranasinghe MG, Zalatimo O, Rizk E, et al. Idiopathic hypertrophic spinal pachymeningitis. J Neurosurg Spine 2011;15(2):195-201

4 Mikawa Y, Watanabe R, Hino Y, Hirano K. Hypertrophic spinal pachymeningitis. Spine 1994;19(5):620-625

5 Pai S, Welsh CT, Patel S, Rumboldt Z. Idiopathic hypertrophic spinal pachymeningitis: report of two cases with typical MR imaging findings. Am J Neuroradiol 2007;28(3):590-592

6 Bosman T, Simonin C, Launay D, Caron S, Destée A, Defebvre L. Idiopathic hypertrophic cranial pachymeningitis treated by oral methotrexate: a case report and review of literature. Rheumatol Int 2008;28(7):713-718

7 Kupersmith MJ, Martin V, Heller G, Shah A, Mitnick HJ. Idiopathic hypertrophic pachymeningitis. Neurology 2004;62(5):686-694

8 Hamilton SR, Smith CH, Lessell S. Idiopathic hypertrophic cranial pachymeningitis. J Clin Neuroophthalmol 1993;13(2):127-134 